\title{
Berberine Improves Cognitive Impairment by Simultaneously Impacting Cerebral Blood Flow and $\beta$-Amyloid Accumulation in an APP/tau/PS1 Mouse Model of Alzheimer's Disease
}

\author{
Chenghui Ye ${ }^{1,+}$, Yubin Liang ${ }^{2,+}$, Ying Chen ${ }^{1}$, Yu Xiong ${ }^{1} \mathbb{D}$, Yingfang She ${ }^{1} \mathbb{D}$, Xiaochun Zhong ${ }^{1}$, Hongda Chen ${ }^{3, *}$ \\ and Min Huang ${ }^{1, *}$
}

\section{check for}

updates

Citation: Ye, C.; Liang, Y.; Chen, Y.; Xiong, Y.; She, Y.; Zhong, X.; Chen, H.; Huang, M. Berberine Improves Cognitive Impairment by Simultaneously Impacting Cerebral Blood Flow and $\beta$-Amyloid Accumulation in an APP/tau/PS1 Mouse Model of Alzheimer's Disease. Cells 2021, 10, 1161. https://doi.org/ 10.3390/cells10051161

Academic Editors: Kunlin Jin and Christoph Englert

Received: 30 March 2021

Accepted: 6 May 2021

Published: 11 May 2021

Publisher's Note: MDPI stays neutral with regard to jurisdictional claims in published maps and institutional affiliations.

Copyright: (c) 2021 by the authors. Licensee MDPI, Basel, Switzerland. This article is an open access article distributed under the terms and conditions of the Creative Commons Attribution (CC BY) license (https:// creativecommons.org/licenses/by/ $4.0 /)$.
1 Department of Neurology, The Seventh Affiliated Hospital, Sun Yat-sen University, Shenzhen 518107, China; yechh@mail.sysu.edu.cn (C.Y.); cheny769@mail.sysu.edu.cn (Y.C.); xiongy69@mail2.sysu.edu.cn (Y.X.); sheyf3@mail.sysu.edu.cn (Y.S.); zhongxch5@mail.sysu.edu.cn (X.Z.)

2 Department of Neurology, Zhuhai People's Hospital (Zhuhai Hospital Affiliated with Jinan University), Zhuhai 519000, China; liangyubin@stu2017.jnu.edu.cn

3 Department of Traditional Chinese Medicine, The Seventh Affiliated Hospital, Sun Yat-sen University, Shenzhen 518107, China

* Correspondence: chenhd23@mail.sysu.edu.cn (H.C.); huangm78@mail.sysu.edu.cn (M.H.)

+ These authors have contributed equally to this work.

\begin{abstract}
Alzheimer's disease (AD) is accompanied by $\beta$-amyloid $(\mathrm{A} \beta)$, neurofibrillary tangles, and neuron cell death, and is one of the most commonly occurring diseases among the elderly. The pathology of $\mathrm{AD}$ is complex, involving $\mathrm{A} \beta$ overproduction and accumulation, tau hyperphosphorylation, and neuronal loss. In addition, chronic cerebral hypoperfusion $(\mathrm{CCH})$ is ubiquitous in the AD patients and plans a pivotal role in triggering and exacerbating the pathophysiological progress of $\mathrm{AD}$. The goal of this study was to investigate the neuroprotective properties of berberine (BBR) and the underlying mechanism. During the study, BBR was administrated to treat the triple-transgenic mouse model of Alzheimer's disease $(3 \times \mathrm{Tg} \mathrm{AD})$. To thoroughly evaluate the effects of the BBR administration, multiple manners were utilized, for instance, 3D arterial spin labeling technique, Morris water maze assay, immunofluorescence staining, TUNEL assay, laser speckle contrast imaging, western blotting, etc. The results showed that BBR ameliorated cognitive deficits in $3 \times \mathrm{Tg}$ AD mice, reduced the $A \beta$ accumulation, inhibited the apoptosis of neurons, promoted the formation of microvessels in the mouse brain by enhancing brain CD31, VEGF, N-cadherin, Ang- 1 . The new vessels promoted by BBR were observed to have a complete structure and perfect function, which in turn promoted the recovery of cerebral blood flow $(\mathrm{CBF})$. In general, berberine is effective to $3 \times \mathrm{Tg}$ $\mathrm{AD}$ mice, has a neuroprotective effect, and is a candidate drug for the multi-target prevention and treatment of AD.
\end{abstract}

Keywords: Alzheimer's disease; BBR; cerebral blood flow; $\beta$-amyloid

\section{Introduction}

Alzheimer's disease (AD) becomes the leading cause of dementia in the elderly. Characterized by chronic progressive neurodegeneration, AD results in progressive cognitive impairment and, ultimately, death. The AD pathology relates to a variety of factors. The most frequent histopathological changes include the formation of neurofibrillary tangles (NFTs) and senile plaque in the brain, accompanied by neurodegeneration $[1,2]$. Previous research on AD did not deeply explore vascular factors. To be more specific, AD and vascular dementia (VD) were treated as two completely distinct diseases, degenerative diseases and vascular diseases [3,4]. However, emerging evidence implies the contribution of chronic cerebral hypoperfusion $(\mathrm{CCH})$ towards the pathogenesis of $\mathrm{AD}$. To date, researchers observe the reduction of $\mathrm{CBF}$ in temporal, parietal and frontal cortices among 
$A D$ patients [5,6]. It is suggested that the reduction of CBF could be a decisive factor in the pathogenesis of cognitive dysfunction.

Although the occurrence and development of AD are related to many factors, such as $\mathrm{A} \beta$ deposition, tau protein hyperphosphorylation, synaptic pathological changes, and mitochondrial function, cerebrovascular factors play an important role in the pathogenesis of AD [7]. Specifically, cerebral microcirculation plays an important role in maintaining the stability of the brain environment and ion homeostasis of neurons, which is the physiological basis of cerebral microvascular in the pathological changes of AD such as nerve fibrosis and plaque formation [8]. The decrease of CBF suggests that the disturbance of microcirculation occurs before the pathological changes of $\mathrm{AD}$ and could be a documented event at early stages [9]. $A \beta$ is the core component of senile plaques. Its neurotoxicity causes the death and degeneration of neurons. Additionally, it delivers negative impacts towards synaptic structure, such as destruction and a decrease in number. The ultimate outcomes are cognitive decline and behavioral abnormalities [10]. On the other side, researchers observe an increase in the synthesis of $\beta$-APP, and significant improvements in $\beta$-secretase BACE1 activity in the brain with the existence of cerebral hypoperfusion [10]. These changes can break the balance between the production and degradation of $A \beta$, and result in an abnormal deposition of $\mathrm{A} \beta$. Another well-accepted pathological feature of $\mathrm{AD}$ is neurofibrillary tangle, which is formed by abnormal phosphorylation of Tau protein [11,12]. The decrease of CBF causes early cell injury, which is supported by a growing number of studies. Subsequently, protein phosphatase activity decreases, and protein kinase activity increases, both of which lead to the hyperphosphorylation of Tau protein [13]. According to latest discoveries, therapies targeting one single protein or pathway have little therapeutic effects on complex diseases, such as AD [14]. Instead, novel efficient therapeutics are more likely to be discovered via targeting several key pathways simultaneously.

Berberine (BBR), also known as berberine hydrochloride, is a natural isoquinoline alkaloid extracted from Rhizoma Coptidis. It has a variety of pharmacological effects, including anti-viral, antibacterial, anti-inflammatory, anti-cancer, hypoglycemic, and lipid regulation $[15,16]$. Additionally, BBR has potential therapeutic effects on $\mathrm{AD}$ through different pathological mechanisms, such as lowering $A \beta$ levels, inhibiting the phosphorylation of Tau protein, anti-oxidation, inhibiting the activity of AchE and MAO, and regulating lipids, hypoglycemic. The studies of BBR regarding its treatment effects towards AD mainly focus on how BBR intervenes with $A \beta$. To be more specific, it illuminates the neurotoxicity of $A \beta$, the inflammatory response induced by $A \beta$, and the production of $A \beta$. However, little attention has been devoted to the impacts of BBR on the CBF of AD.

Therefore, in the present study, we aimed to investigate the effects of $B B R$ on $A D$ regarding the changes in $\mathrm{CBF}$, and the following impacts towards the cognitive function. Given this focus, a novel AD mouse model was utilized.

\section{Materials and Methods}

\subsection{Drugs and Reagents}

Both BBR (99\% purity) for treatment and dimethyl sulfoxide (DMSO) were purchased from Sigma-Aldrich (St. Louis, MO, USA). Antibodies specific to A $\beta 1-42$, DAPI, GFAP, NeuN, caspase-3, CD31, VEGF, N-cadherin, Ang-1 and GAPDH were purchased from Abcam (Cambridge, UK). In situ cell death detection kits were utilized to perform the TdT-mediated dUTPnick-end labeling (TUNEL). All other reagents used in the experiment were reagent-grade. Table 1 shows the antibody information. 
Table 1. Antibody information.

\begin{tabular}{ccccc}
\hline Antibody & Host & Application & Source & Dilutions \\
\hline A $\beta$ & Rabbit & WB/IF & Abcam & $1: 500$ \\
GFAP & Rabbit & WB/IF & Abcam & $1: 500$ \\
NeuN & Rabbit & WB/IF & Abcam & $1: 500$ \\
CD31 & Rabbit & WB/IF & Abcam & $1: 500$ \\
VEGF & Rabbit & WB & Abcam & $1: 500$ \\
GAPDH & Rabbit & WB & Abcam & $1: 2500$ \\
N-cadherin & Rabbit & WB & Cell signaling & $1: 1000$ \\
Ang-1 & Rabbit & WB & Cell signaling & $1: 1000$ \\
caspase3 & Rabbit & WB/IF & Cell signaling & $1: 1000$ \\
\hline
\end{tabular}

WB: Western blot blatting; IF: immunofluorescence.

\subsection{Animals and Treatment}

The $3 \times \mathrm{Tg}$ AD mice were purchased from Jackson Laboratory (Bar Harbor, ME, USA) to complete the experimental study by expressing human mutant genes, including APPswe, PS1M146V, and tauP301L. A $\beta$ was detected in the cells of these six-month-old mice [17]. In the treatment group (BBR $+3 \times \mathrm{Tg})(n=12$; male), drinking water containing $100 \mathrm{mg} / \mathrm{kg} /$ day berberine was given from four months old for four months until they developed cognitive impairment and typical pathological features. The dosage of berberine in this experiment is based on the previous research report [18]. The other two groups were treated with conventional drinking water without any other addition. The two groups were $3 \times \operatorname{Tg}$ AD mice $(3 \times \operatorname{Tg})(n=12$; male) and male non-transgenic wild-type (WT) mice $(n=12)$. The three groups were all carried out under the same standard laboratory conditions, with a temperature of $22 \pm 2{ }^{\circ} \mathrm{C}$ and a light/dark cycle of $12 \mathrm{~h}$, and free drinking water and eating. Each cage contained three or four objects. The purpose of this experiment was to minimize the pain suffered by animals, which is reflected in all aspects of the experiment process, such as reducing the number of animals used and using the substitute methods of in vivo technology when feasible. This study has always followed the Animal Care and Institutional Ethical Guidelines in China.

\subsection{Magnetic Resonance Imaging}

\subsubsection{Scanning Parameters}

The MRI was from GE Healthcare, Milwaukee, Wisconsin, USA, with an eight-channel wrist coil discovery 750 3.0T scanner (GE Healthcare, Milwaukee, Wisconsin, USA). All mice were scanned with the same parameters. Each mouse was anesthetized with $10 \%$ chloral hydrate $(0.3 \mathrm{~mL} / 100 \mathrm{~g})$ and placed in a supine position, then scanned. In this study, all imaging parameters of 3D ASL series are the same as those reported in previous studies [19]. In other words, 15 slices are scanned in ascending order with slice thickness $=4 \mathrm{~mm}$ without gaps. Other core parameters include: field of view $=120 \mathrm{~mm} \times 120 \mathrm{~mm}$, matrix $=512$ (points) $\times 12$ (arms); number of excitations $=5$, bandwidth $=62.5 \mathrm{kHz}$, scan duration was $9 \min 14 \mathrm{~s}$, labeling duration $=1650 \mathrm{~ms}$, post-labeling delay $=1025 \mathrm{~ms}$, repetition time $=4132 \mathrm{~ms}$ and echo time $=11 \mathrm{~ms}$. In addition, MR imaging of the blood vessels in mice was obtained by time-of-flight MR angiography (TOF MRA) and 3D Fast SPGR. The scanning parameters of TOF MRA are as follows: echo time $=3.9 \mathrm{~ms}$, repetition time $=20 \mathrm{~ms}$, field of view $=80 \mathrm{~mm} \times 60 \mathrm{~mm}$, matrix $=320 \times 224$, number of excitations $=1$, bandwidth $=31.2 \mathrm{kHz}$, and scan duration was $231 \mathrm{~s}$.

\subsubsection{D Arterial Spin Labeling Technique}

CBF in different brain regions of the mice was measured using the 3D arterial spin labeling (ASL) technique. The processes and the parameters of this technique were kept the same as the previous study [19]. 


\subsection{Morris Water Maze Task}

The learning and memory abilities of all mice were evaluated by a five-day (d) Morris water maze task at the age of eight months [20]. During the five-day evaluation, all mice in the three groups received the same treatment as before. All the equipment and test procedures in the experiment were consistent with the previous research [21]. Specifically, this experiment had a round white metal pool; its diameter was $160 \mathrm{~cm}$ and its height was $50 \mathrm{~cm}$. The pool was filled with water of constant temperature $\left(22 \pm 1^{\circ} \mathrm{C}\right)$ to $26 \mathrm{~cm}$ deep. Then, the water maze software (Tianyancha, Sichuan, China) divided the pool into four quadrants and was equipped with a translucent acrylic platform. The platform was placed in the center of the northwest quadrant and 1 to $2 \mathrm{~cm}$ below the water surface, with a diameter of $12 \mathrm{~cm}$ and a height of $24 \mathrm{~cm}$.

\subsubsection{Spatial Learning Test}

All mice were tested for four consecutive experiments and five days of spatial learning. The starting position of the mice was selected in the four quadrants of the pool edge and changed every day, but the position of the platform remained unchanged. In the first part of each experiment, the mice were gently placed in the water with their noses toward the surface, at different starting points (north, south, east, and west) from the wall. It took up to $60 \mathrm{~s}$ for each mouse to find the hidden platform. If the escape platform is not found within $60 \mathrm{~s}$, it takes another $30 \mathrm{~s}$ to manually guide it to the platform. The escape traces of all mice can be recorded by a camera mounted on the ceiling directly above the pool. The human visual system (HVS) water maze program was used for subsequent analysis of escape latency (Water Maze 3, Actimetrics, Evanston, IL, USA). The researchers did not know the group allocation of each mouse during the experiment; that is, all the experiments were carried out by blind method.

\subsubsection{Probe Trial}

Short- and long-term memory integration was evaluated by probe test. The experiment was carried out after the last experiment, at $24 \mathrm{~h}$ and $72 \mathrm{~h}$, respectively. At the beginning of the experiment, the platform was removed, and then the mice were placed in the quarter quadrant of the pool, which was the position of the previous platform. In each probe test, each mouse had a swimming time of $60 \mathrm{~s}$. The time spent in the quadrant pre-placed with the platform and the time spent in the platform position were recorded to evaluate the short- and long-term memory of the mice.

\subsection{Immunofluorescence Staining}

The vector paraffin slices of the mouse brain were mounted on a $5 \mathrm{~mm}$-thick slide. The slices were pre-treated at a high temperature of $0.01 \mathrm{~mol} / \mathrm{L}$ citrate buffer $(\mathrm{pH}=6.0)$ for $5 \mathrm{~min}$, then sealed off in PBS with 5\% goat serum for $10 \mathrm{~min}$. These slices were further incubated overnight at $4{ }^{\circ} \mathrm{C}$ with an anti-resistance. The slices were then sliced with a secondary resistance (1:500 in PBS) and incubated for $1 \mathrm{~h}$ at $37^{\circ} \mathrm{C}$.

A $31-42$, GFAP, NeuN, caspase-3 and CD31 were detected by the second antibody (anti-mouse and anti-rabbit; Alexa Fluor 488 and 695, (Multi Sciences Biotech, Hangzhou, China)) coupled with Alexa fluor fluorescent dye. For each mouse, three equidistant parts were evaluated to cover the entire hippocome. To analyze and quantify immune response areas, these slices were imaged using a fluorescent microscope (Olympus, Japan) and analyzed using ImagePro Plus 6.0 software (Media Cybernetics, Shanghai, China). 


\subsection{TUNEL Assay}

An in situ pod cell death detection kit (Roche) was used to detect cell death in brain tissue. In other words, the assay determines apoptosis by detecting DNA breaks caused by apoptotic signal cascades and DNA strand breaks labeled with terminal deoxynucleotidyl transferase.

\subsection{Laser Speckle Contrast Imaging}

Real-time 2D CBF perfusion information was provided by a laser speckle contrast imaging system. Before the experiment, the mice were sedated and hypnotized. A median longitudinal incision was made at the back of the brain, and the skin was pulled to the sides to expose the skull. Then, the skull was placed $10 \mathrm{~cm}$ below the scan head to record CBF perfusion in real time.

\subsection{Western Blot Assay}

Before moving onto assays on the brain tissue, all tissue samples were required to be homogenized with lysis buffer plus $1 \mathrm{mM}$ PMSF and protease inhibitor cocktail. A BCA protein assay kit was used to assess protein concentration. The total protein extract from each sample was equal, which was $20 \mu \mathrm{g}$ per well. These protein extracts were separated by SDS-PAGE and transferred to polyvinylidene fluoride (PVDF) membranes. Five percent fatfree milk was utilized as the blocking agents. Afterwards, CD31, VEGF, N-cadherin, Ang-1 and GAPDH were probed with the corresponding primary antibodies (1:1000), followed by the incubation with HRP-conjugated anti-rabbit antibody or HRP-anti-mouse antibody. These blots were developed using ECL detection reagent and visualized as Kodak Image Station 4000 mm (Carestream Health Inc., New Haven, CT, USA). Quantitative software (National Institutes of Health, Bethesda, MD, USA) was used to quantify the characteristics of all frequency bands.

\subsection{Statistical Analysis}

The statistical analysis was carried out using Statistical Product and Service Solutions (SPSS) 17.0 software (SPSS Inc., Chicago, IL, USA). Comparison between groups was analyzed by one-way ANOVA test followed by post-hoc test (least significant difference). $p<0.05$ was set to be statistically significant.

\section{Results}

\subsection{BBR Accelerates the Recovery of CBF in the Brain of $3 \times \mathrm{Tg}$ AD Mice}

The 3D ASL technique was applied to detected CBF in every mouse across the three groups. The color signals in the image, ranging from green to red, represent an incremental scale of CBF level. Compared with WT group, most areas in $3 \times \mathrm{Tg}$ AD group demonstrated blue or green, suggesting an evident lower $\mathrm{CBF}$ in brain in $3 \times \mathrm{Tg}$ AD group. The BBR treatment group $(\mathrm{BBR}+3 \times \mathrm{Tg}$ ) showed a relatively higher $\mathrm{CBF}$ after four months of administration, compared with the $3 \times \mathrm{Tg}$ AD group of the same age. The dynamic changes in $\mathrm{CBF}$ across different group were further analyzed in a quantitative manner. This difference between the BBR treatment group and $3 \times \mathrm{Tg}$ AD group was statistically significant (Figure $1 \mathrm{C}, \mathrm{D}$ ). Between the WT group and $3 \times \mathrm{Tg}$ AD group, the latter showed a significantly lower CBF (Figure 1D, $p<0.01$ ). In the comparison between the BBR $+3 \times \mathrm{Tg} A D$ group and $3 \times \mathrm{Tg} \mathrm{AD}$ group, the treatment group demonstrated a significantly higher $\mathrm{CBF}$ (Figure 1D, $p<0.01$ ). It supports the hypothesis that BBR could accelerate the recovery of $\mathrm{CBF}$ in a $3 \times \mathrm{Tg} \mathrm{AD}$ mouse model. 


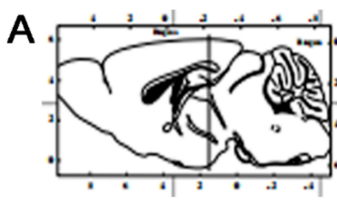

B
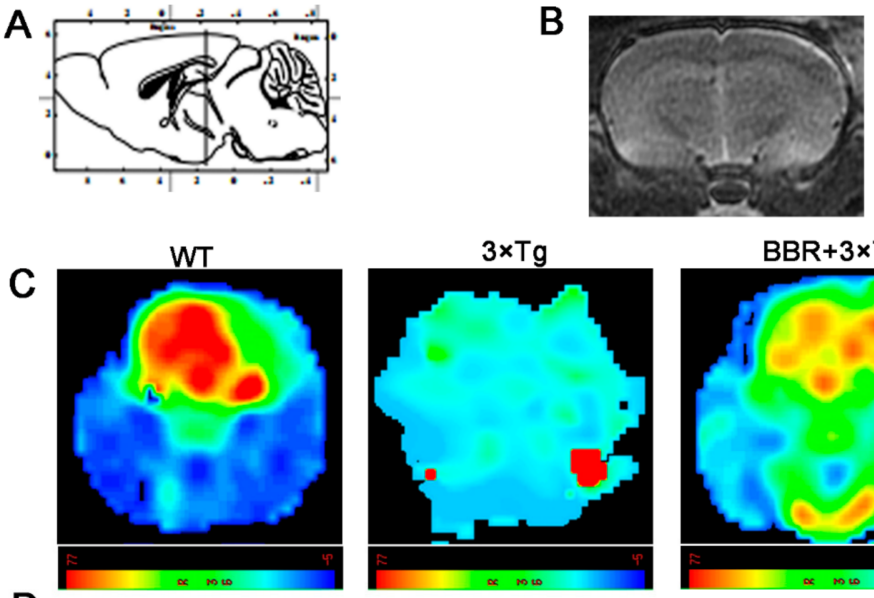

$\mathrm{BBR}+3 \times \mathrm{Tg}$

D

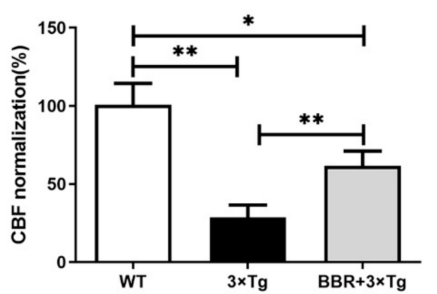

Figure 1. Magnetic resonance imaging (MRI; 3D ASL) analysis showing changes in CBF in the brain of mice. (A) A cross-sectional image corresponding to the brain mapping of mice. (B) The gray-scale images are T2 imaging. (C) MRI images show the changes in CBF in the brain of mice, and color images are those with 3D ASL. The red label indicates a relatively higher CBF level, while the green indicates a relatively lower CBF level. (D) Quantitative analysis results of CBF in mice brains. WT, wild-type mice, $3 \times \mathrm{Tg}, 3 \times \mathrm{Tg} \mathrm{AD}$ mice, $\mathrm{BBR}+3 \times \mathrm{Tg} ; 3 \times \mathrm{Tg}$ AD mice were given $\mathrm{BBR}$ at $100 \mathrm{mg} / \mathrm{kg} /$ day. One-way ANOVA and Dunnett's post-hoc test were used to analyze all the data, expressed as mean $\pm \mathrm{SD} ; n=12$ animals/group. ${ }^{*} p<0.05,{ }^{* *} p<0.01$.

\subsection{BBR Relieves Cognitive Impairments in $3 \times T g$ AD Mice}

The Morris water maze test assessed spatial learning by measuring the time each mouse spent to find the hidden platform (also known as escape latency). The length of escape latency is supposed to be negatively correlated with the level of spatial learning ability. Additionally, in post hoc multiple comparisons, no significant differences were found in the swimming speed of all groups (Figure 2B, $p>0.05$ ). Thus, the results from the Morris water maze test could solidly reflect the effect of BBR on memory impairment in $3 \times \mathrm{Tg} \mathrm{AD}$ mice. In a five-day training trial, compared with WT mice, $3 \times \mathrm{Tg}$ AD mice exhibited a significantly longer escape path and remarkably longer escape latency to find the platform (Figure 2A). The escape latency of the BBR-treated $3 \times \mathrm{Tg} \mathrm{AD}$ mice was significantly shorter than that in $3 \times \mathrm{Tg}$ AD mice (Figure 2A). According to the probe trial assay results, the platform span of $3 \times \mathrm{Tg} \mathrm{AD}$ mice treated with $\mathrm{BBR}$ was shorter than that of $3 \times \mathrm{Tg}$ AD mice (Figure 2C,D). Moreover, comparison of the swimming tracts across the three groups suggests that tracts from the BBR treated $3 \times \mathrm{Tg} \mathrm{AD}$ mice group match with those from the WT group better than the $3 \times \mathrm{Tg} \mathrm{AD}$ group (Figure $2 \mathrm{E}$ ). 
A

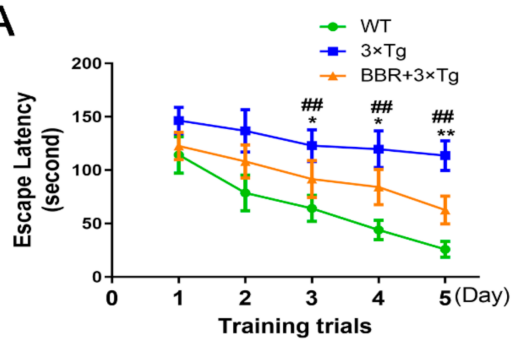

C

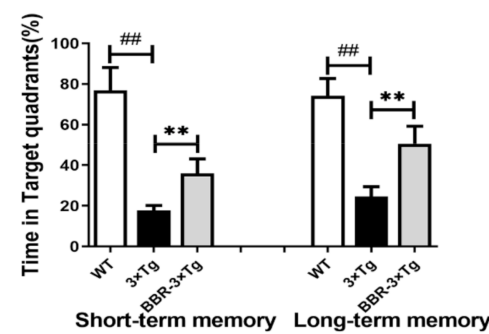

B

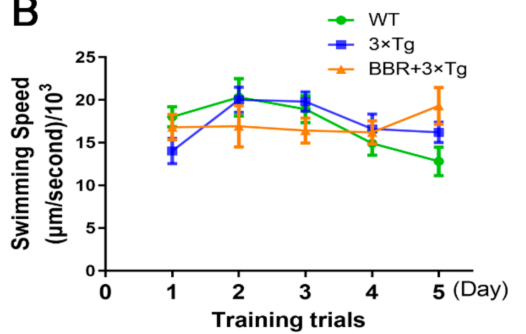

D

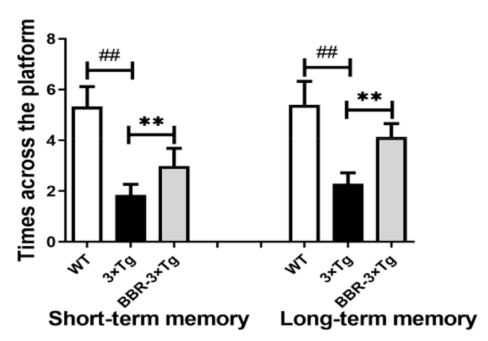

$\mathrm{BBR}+3 \times \mathrm{Tg}$

E
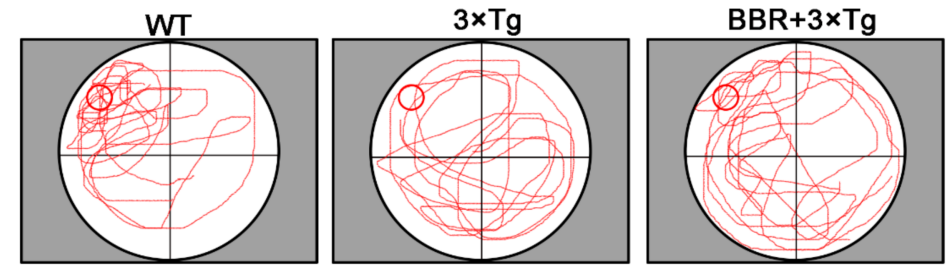

Figure 2. A Morris water maze task was used to evaluate the effect of berberine on the spatial learning and memory of $3 \times \mathrm{Tg} \mathrm{AD}$ mice. (A) In five-day training trial, the escape latency of each mouse was measured. (B) There were no significant differences in swimming speed across the three groups. (C) In the probe trail, the frequency at which the mouse passed through the submerged platform placement area during the training trial was recorded. (D) Time spent searching for the pre-placed platform in the target quadrant, in both the short- and long-term memory tests. (E) The swimming tracks of the mice from the three groups made in the water tank on the last day of the test. WT, wild-type mice, $3 \times \mathrm{Tg}, 3 \times \mathrm{Tg} \mathrm{AD}$ mice; $\mathrm{BBR}+3 \times \mathrm{Tg} ; 3 \times \mathrm{Tg} \mathrm{AD}$ mice were given $\mathrm{BBR}$ at $100 \mathrm{mg} / \mathrm{kg} /$ day. One-way ANOVA and Dunnett's post-hoc test were used to analyze all the data, which are presented as mean $\pm \mathrm{SD} ; n=12$ animals/group. ${ }^{*} p<0.05,{ }^{\# \#} p<0.01,3 \times \mathrm{Tg}$ AD group vs. WT group. ${ }^{*} p<0.05,{ }^{* *} p<0.01$, BBR treated $3 \times \mathrm{Tg}$ vs. $3 \times \mathrm{Tg}$ group.

\subsection{BBR Reduces the Production of $A \beta$ and Inhibits Apoptosis in the Brains of $3 \times \mathrm{Tg} A D$ Mice}

The effect of BBR on $\mathrm{A} \beta$ burden in the brain was also investigated in the $3 \times \mathrm{Tg} \mathrm{AD}$ mice. The results of immunofluorescent staining of $\beta$-amyloid in the hippocampus of mice from all three groups were shown in Figure 3A. As expected, there was little aggregation of $\beta$-amyloid found in the WT mice, while the $\beta$-amyloid aggregation was apparent in the $3 \times \mathrm{Tg} \mathrm{AD}$ and $\mathrm{BBR}$-treated $3 \times \mathrm{Tg} \mathrm{AD}$ groups (Figure $3 \mathrm{~A}$ ). Moreover, between the latter two groups, the density of $\beta$-amyloid aggregating in the hippocampus was remarkably higher in the $3 \times \mathrm{Tg} \mathrm{AD}$ group, and the labeling for $\beta$-amyloid was also more intense (Figure $3 \mathrm{~B}$, $p<0.01)$. 


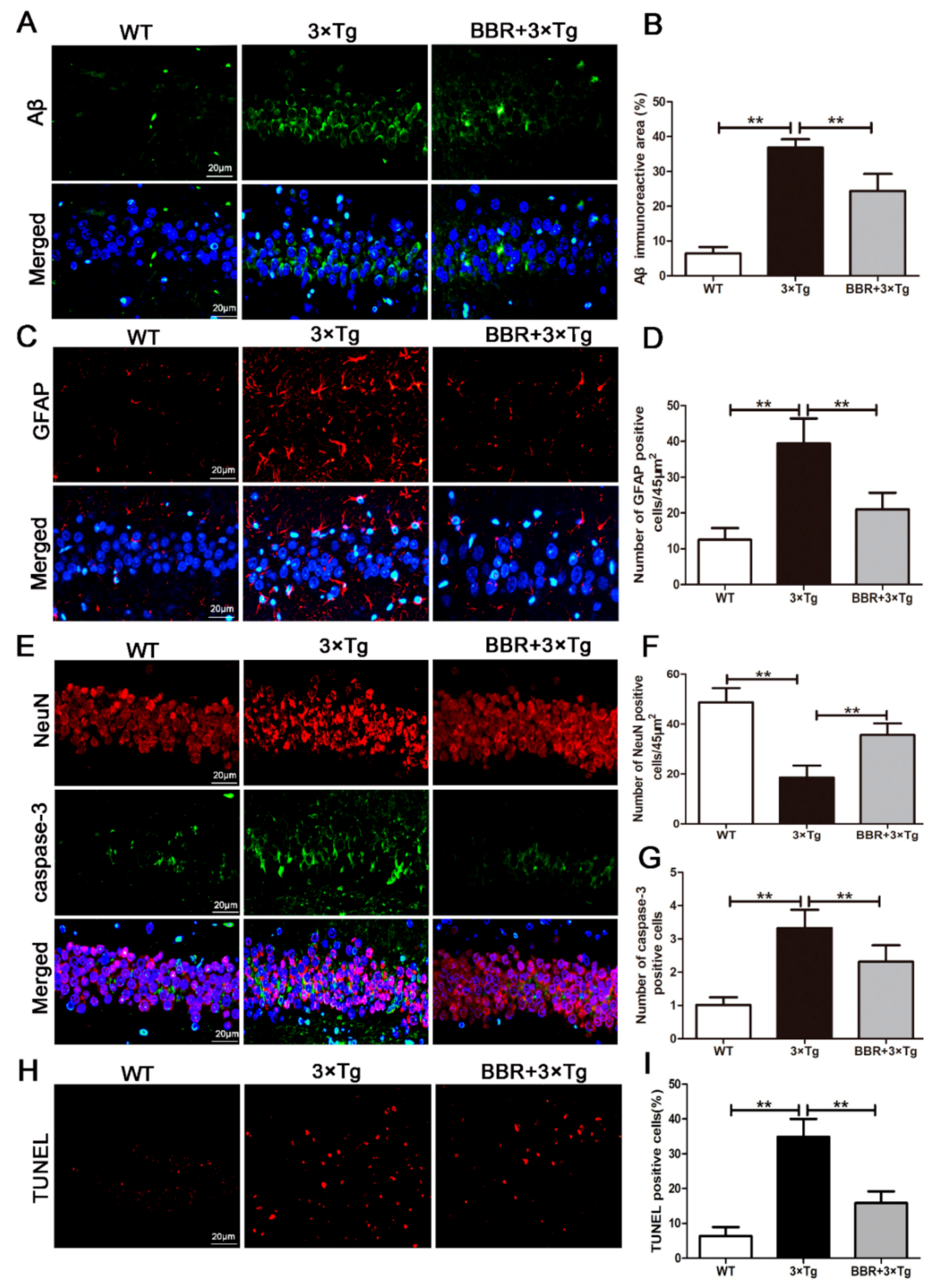

Figure 3. BBR reduced the production of $A \beta$ and inhibited apoptosis in the brains of $3 \times \operatorname{Tg} A D$ mice. (A) Immunofluorescence of $A \beta$ (green) in the hippocampi of mice from the three groups. Scale bar: $20 \mu \mathrm{m}$. (B) Quantification of $A \beta$ immunoreactivity in the hippocampi of mice. (C) Immunofluorescence of GFAP (red) in the hippocampi of mice from the three groups. Scale bar: $20 \mu \mathrm{m}$. (D) Quantification of GFAP immunoreactivity in the hippocampi of mice. (E) Immunofluorescence of NeuN (red) and Caspase-3 (green) in the hippocampi of mice from the three groups. Scale bar: $20 \mu \mathrm{m}$. (F) Quantification of NeuN and Caspase-3 immunoreactivity in the hippocampi of mice. (G) Quantification of Caspase-3 immunoreactivity in the hippocampi of mice. (I) The number of TUNEL-positive neurons in each group was averaged. $(\mathbf{H})$ The number of TUNEL-positive neurons (red). Scale bar: $20 \mu \mathrm{m}$. WT, wild-type mice; $3 \times \mathrm{Tg}$, $3 \times \mathrm{Tg}$ AD mice; $\mathrm{BBR}+3 \times \mathrm{Tg}$; $3 \times \mathrm{Tg}$ AD mice were given BBR at $100 \mathrm{mg} / \mathrm{kg} /$ day. One-way ANOVA and Dunnett's post-hoc test were used to analyze all the data, which are presented as mean $\pm \mathrm{SD} ; n=12$ animals/group. ${ }^{* *} p<0.01$.

Immunofluorescence was applied to examine the number of GFAP in the hippocampus of each group. Based on the outcomes shown in Figure 3C, a small number of GFAP were observed in the hippocampus of the WT group. Nevertheless, a noticeable amount of GFAP were observed in the hippocampus of the $3 \times \mathrm{Tg}$ AD group, with no BBR administration. The amount of GFAP was significantly lower in the $3 \times$ Tg AD group with BBR administration, compared with the $3 \times \mathrm{Tg}$ AD group without administration (Figure 3D, $p<0.01$ ). Neuronal apoptosis in hippocampus was also taken into consideration. Immunofluorescence was 
used to evaluate the apoptosis level, via detecting the expression of NeuN, Caspase-3 and TUNEL. Compared with the WT group, a significant increase in the expression of caspase- 3 in the hippocampus of the $3 \times \mathrm{Tg}$ AD group, as well as a meaningful decrease in the expression of NeuN was observed (Figure $3 \mathrm{E}, p<0.01$ ). In line with the previous findings, $\mathrm{BBR}$ administration relieved these changes. In the BBR-treated $3 \times \mathrm{Tg} \mathrm{AD}$ group, compared with the $3 \times \mathrm{Tg}$ AD group, the expression of caspaes- 3 in the hippocampus was significantly lower (Figure 3G, $p<0.01$ ), and the expression of NeuN was significantly higher (Figure 3F, $p<0.01$ ), both of which were closer to the level observed in WT group. As shown in Figure $3 \mathrm{H}$, the number of TUNEL-positive cells in the brain tissue of $3 \times \mathrm{Tg}$ $\mathrm{AD}$ mice was larger than that of the WT mice (Figure $3 \mathrm{H}, p<0.01$ ). Merely considering the two $3 \times \mathrm{Tg} \mathrm{AD}$ groups, with and without BBR treatment, the treatment group demonstrated a significantly less TUNEL-positive cells (Figure 3I, $p<0.01$ ) in the brain.

3.4. BBR Increases the Cerebral CBF Perfusion and Proangiogenic Factors in the Brains of $3 \times \mathrm{Tg}$ $A D$ Mice

The cerebral CBF perfusion was detected via laser speckle contrast imaging. According to the laser speckle contrast imaging, the CBF perfusion in the WT mice group was obviously higher than that in $3 \times \mathrm{Tg} \mathrm{AD}$ mice group (Figure $4 \mathrm{~A}$ ). The difference was statistically significant. Compared with the $3 \times \mathrm{Tg} \mathrm{AD}$ mice group, $\mathrm{CBF}$ in the BBR-treatment $3 \times \mathrm{Tg}$ AD group was significantly higher (Figure $4 \mathrm{~B}, p<0.01$ ). It was well-indicated that BBR treatment could increase the blood perfusion in brain tissue. It can be inferred that BBR promotes the formation of blood vessels, and the new vessels have blood flow through, and it is functional blood.
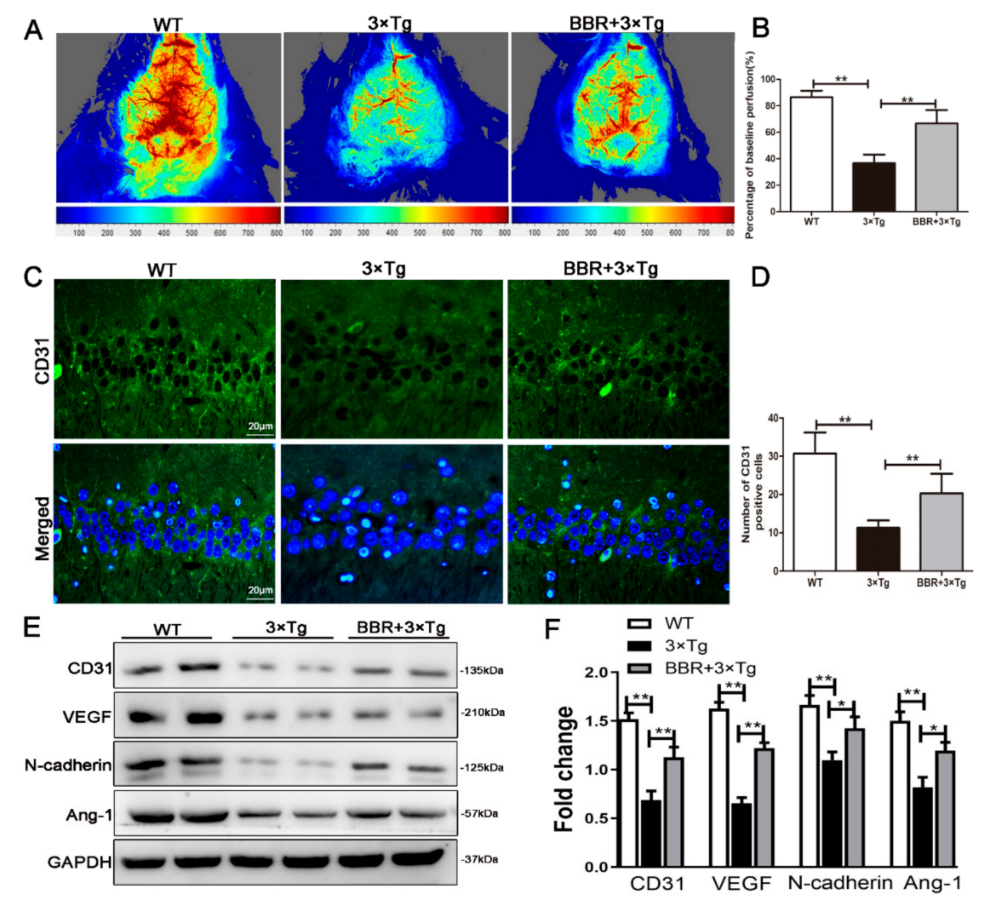

Figure 4. BBR increased the cerebral cortical blood flow perfusion and increased proangiogenic factors in the brains of $3 \times \mathrm{Tg}$ AD mice. (A) Representative images of cerebral cortical blood flow perfusion in three groups. (B) Analysis of the level of cerebral cortical blood flow perfusion in three groups. (C) Immunofluorescence of CD31 (green) in the hippocampi of mice from the three groups. Scale bar: $20 \mu \mathrm{m}$. (D) Quantification of CD31 immunoreactivity in the hippocampi of mice. (E) Western blotting showed protein level of CD31, VEGF, N-Cadherin and Ang-1 in three groups. (F) Analysis of protein level of CD31, VEGF, N-Cadherin and Ang-1. WT, wild-type mice; $3 \times \mathrm{Tg}$, $3 \times \mathrm{Tg}$ AD mice; $\mathrm{BBR}+3 \times \mathrm{Tg} ; 3 \times \mathrm{Tg}$ AD mice were given $\mathrm{BBR}$ at $100 \mathrm{mg} / \mathrm{kg} /$ day. One-way ANOVA and Dunnett's post-hoc test were used to analyze all the data, which are presented as mean $\pm \mathrm{SD}$; $n=12$ animals/group. ${ }^{*} p<0.05,{ }^{* *} p<0.01$. 
CD31 plays an important role in the formation of blood vessels. It can also be used as a manner to quantitatively evaluate the role of angiogenesis factors in angiogenesis. As a result, it has become an indicator of common angiogenesis in clinical practice [22]. We tested the number of CD31 to determine whether there was angiogenesis or not. Figure $4 \mathrm{C}$ shows the number of CD31-positive staining. It demonstrated that staining in $3 \times \mathrm{Tg} \mathrm{AD}$ group was significantly lower than that in WT group (Figure $4 \mathrm{D}, p<0.01$ ). Compared with $3 \times \mathrm{Tg}$ AD group, the quantity of CD31-positive staining in the hippocampus increased significantly in the BBR-treated group (Figure 4D, $p<0.01$ ). Western-blot was used to detect the expression of CD31, VEGF, Ang-1 and N-Cadherin in the hippocampus of mice, which was further used to evaluate angiogenesis in the hippocampus (Figure 4E). Compared with WT group, the expression of CD31, VEGF, Ang-1 and N-cadherin in the hippocampus of $3 \times \mathrm{Tg}$ AD group was significantly decreased (Figure $4 \mathrm{~F}, p<0.01$ ). Comparing the outcomes between $3 \times \mathrm{Tg}$ AD group and BBR $+3 \times \mathrm{Tg}$ AD group, the latter exhibited significantly higher levels in the expressions of CD31, VEGF, Ang-1 and N-cadherin in the hippocampus (Figure $4 \mathrm{~F}, p<0.01$ ).

\section{Discussion}

Although BBR is a well-known neuroprotective agent $[15,16]$, few studies have focused on whether BBR can promote the recovery of CBF in AD mice. In this experiment, we used a $3 \times \mathrm{Tg}$ AD mouse model and gave a four-month BBR administration to the treatment $3 \times \mathrm{Tg}$ AD mice group. We used the ASL scanning method, Morris water maze test, immunofluorescence assay, TUNEL, western blotting and laser speckle contrast imaging to conduct a thorough evaluation of how BBR impacts the cognitive function of $3 \times \mathrm{Tg} A D$ mice, and which pathways were involved. The outcomes of our study suggest that BBR has a beneficial effect in Alzheimer's disease via different mechanisms. First, BBR ameliorates cognitive deficits in the $3 \times \mathrm{Tg}$ mouse model of Alzheimer's disease. Second, BBR not only decelerates the production of $A \beta$, but also inhibits the apoptosis of neurons. Last but not least, BBR plays a positive role via promoting the formation of microvessels in the mouse brain, which leads to the recovery of $\mathrm{CBF}$ in $3 \times \mathrm{Tg}$ AD mice.

Research from various aspects, including epidemiology, clinical medicine, neuropathology, throw more light on the correlation between the reduction of blood flow in the brain and the pathogenesis of cognitive dysfunction [3,23]. From the perspective of medical imaging, studies also provide solid evidence that AD has insufficient perfusion in the temporal parietal (including hippocampus), hippocampus [24], and posterior cingulate gyrus [25]. The level of blood flow level in the hippocampus is recognized as the basis for the diagnosis of early AD [26]. Proved by one previous study, after 25 weeks, the mice's bilateral common carotid arteries being ligated, chronic cerebral ischemia could cause $\beta$ APP cleavage to form $A \beta$ fragment. Other observed consequences include extracellular amyloid deposition, as well as some other pathological changes similar to AD [27]. More importantly, these known patterns formulate a vicious loop, which remarkably speeds up the pathological process. $A \beta$, deposited on the basement membrane of capillaries and perivascular drainage arteries, enhances the endothelial contracture function secreted by endothelial cells. It contributes to cerebral vasoconstriction, further aggravating hypoperfusion. This secondary hypoperfusion accelerates the deposition of $A \beta$ [11]. In conclusion, both chronic hypoperfusion and deposition of $\mathrm{A} \beta$ represent causal factors and reinforce each other. They simultaneously accelerate the pathological process of AD together.

With a long history of being used as a monomer in traditional Chinese medicine, BBR is recognized as safe for clinical use. Research has proven its beneficial impact to decrease the CBF and relieve cognitive deficits in rats with chronic cerebral hypoperfusion $[28,29]$. In our study, we explored if BBR had a similar effect on $\mathrm{CBF}$ and behavioral deficits in $3 \times \mathrm{Tg}$ AD mice. The utilized AD mouse model showed a significant decrease of $\mathrm{CBF}$, which is slowly progressive, compared to the WT group. The observed CBF decrease was partly recovered by BBR administration according to the comparison between $3 \times \mathrm{Tg}$ $\mathrm{AD}$ mice and $\mathrm{BBR}+3 \times \mathrm{Tg} \mathrm{AD}$ mice (Figure 1 ). Additionally, analysis of behavior tests 
showed better cognitive performance in BBR treatment $3 \times \mathrm{Tg}$ AD mice at eight months (Figure 2). It suggests that BBR has potent influences on improving CBF and cognitive deficits in $3 \times \mathrm{Tg} \mathrm{AD}$ mice.

Next, we explored the effect of BBR administration on $A \beta$ and apoptosis changes among $3 \times \mathrm{Tg}$ AD mice. It is well-established that $\mathrm{A} \beta$ has neurotoxic effects, which contributes to neuronal degeneration, death, apoptosis, synaptic destruction and number reduction [30]. The eventual outcomes include cognitive decline and behavioral abnormalities [10]. In accordance with previous research, our findings suggest significant increases in GFAP and neuronal loss, as well as a significant decrease in NeuN in the brain of $3 \times \mathrm{Tg}$ AD mice (Figure 3). In the pathological state, Astrocyte AS is activated by $A \beta$ and other toxic substances, which can produce cytoinitis factors, complements, oxygen free radicals, etc. and initiate inflammatory reactions, promote the damage and death of nerve cells, and aggravate the process of $\mathrm{AD}$ [31]. Astrocyte pathological changes can cause damage to vascular regulation and small arterial contraction and hypoxia of tissues, which play an important role in the pathogenesis of AD [32]. The experimental study showed that compared with the WT group, the expression of GFAP in the hippocampi of the $3 \times \mathrm{Tg}$ $\mathrm{AD}$ group increased significantly, indicating that the damage to hippocup tissue in the $3 \times \mathrm{Tg}$ AD group was obvious, the ascension was active compared with the $3 \times \mathrm{Tg} \mathrm{AD}$ group, and the expression of hippocome GFAP in the berberine group was significantly reduced, indicating that the berberine group inhibited the excessive growth of AD mice AS. This may be related to the improvement of local blood supply by berberine, reducing the toxicity of $A \beta$, and thus repairing damage to nerve vascular units. It is probably due to the neurotoxic effects of $A \beta$. Our study supports that BBR has a strong neuroprotection on ameliorating neuronal loss in $3 \times \mathrm{Tg} \mathrm{AD}$ mice at 9 months (Figure 3). Meanwhile, BBR reduces $A \beta$ production and relieves cell apoptosis.

The decrease of $C B F$ and $A \beta$ deposition reinforce each other and accelerate the pathological process of AD [11]. Therefore, we posited that the beneficial role of BBR towards neuronal loss and neurodegeneration in $3 \times \mathrm{Tg} \mathrm{AD}$ mice also related to microvascular formation in the brain.

To verify the above hypothesis, we examined the effect of BBR on CD31, Ang-1, VEGF, N-cadherin expression in $3 \times \mathrm{Tg}$ AD mice at eight months. CD31 plays an important role in angiogenesis and can be the specific marker for the identification of vascular endothelial cells. It could also provide quantitative assessment of vascular correction factors in angiogenesis [24]. CD31 is proved to have the capacity of formulating micro vessels and establishing collateral circulation in the mouse brain. Another key function of CD31 is that it facilitates the repair of nerve cells [33]. According to our results, after the administration of $\mathrm{BBR}$ in $3 \times \mathrm{Tg} \mathrm{AD}$ mice, the expression of $\mathrm{CD} 31$ was significantly increased. It implies that BBR is able to induce angiogenesis and strengthen the nutritional supports towards the brain. Vascular endothelial growth factor (VEGF) can directly act on neurons in the central nervous system. The interacting mechanism could be taken in multiple forms, for examples, neurotrophic, neuroprotective, anti-apoptotic, or in the process of cell proliferation. Similar with the CD31, the level of VEGF is relatively lower in $\mathrm{AD}$ patients compared with the healthy population. Such change delivers inhibitory effects on cerebral angiogenesis, neuronal protection, cerebral microvascular trophic factors, and permeability, which in turn aggravates the damage of the brain with the existence of hypoxia [34]. The impact of VEFG also reflects the pathological progress of AD. A negative correlation between the level of VEGF and the development progress of AD's pathological process has been observed [35]. VEGF can act directly on many types of nerve cells to play a neurotrophic and neuroprotective role, enhance cell activity and survival, and promote axon regeneration. The experimental study shows that compared with the WT group, the expression of VEGF in the hippocampi of the $3 \times \mathrm{Tg} \mathrm{AD}$ group mice was significantly decreased, indicating that $3 \times \mathrm{Tg} \mathrm{AD}$ group mice have vascular damage and neovascular regeneration disorders, thus leading to nerve vascular unit damage and brain vascular reserves, poor microcirculation blood supply, and thus cognitive dysfunction, which may 
be related to the nerve and vascular toxicity of $\mathrm{A} \beta$. Compared with the $3 \times \operatorname{Tg} \mathrm{AD}$ group, the VEGF in the Berberine group was significantly increased, which was related to berberinepromoting angiogenesis and the acceleration of the removal of toxic substances such as $A \beta$ by the tissue structure around microvascular vessels. Moreover, angiogenesis is necessary for local tissue metabolism and functional recovery, which can increase cerebral blood flow and improve learning and memory ability. Another factor taken into consideration is Ang-1. Ang-1 is mainly produced by pericytes. It plays a positive role in angiogenesis by binding to the Tie-2 reception on the endothelium [36]. In addition to ensuring the survival of endothelial cells, Ang-1 promotes the maturation of new vessels [37]. It was found in this experiment that the expression of Ang- 1 and $\mathrm{N}$-cadherin were meaningfully higher in $3 \times \mathrm{Tg} \mathrm{AD}$ mice at eight months after treatment with $\mathrm{BBR}$, compared to $3 \times \mathrm{Tg} \mathrm{AD}$ mice with no BBR treatment. $\mathrm{N}$-cadherin is another substance facilitating vascular maturation, realized by promoting the encapsulation of pericytes on endothelial cells [38]. This finding suggests that BBR not only promotes angiogenesis but also facilitates the maturation of new vessels. The latest research finds N-cadherin as a key signaling protein which has the potential to open up the anti-apoptotic pathway of nerve cells [39]. According to the immunofluorescence results of CD31, the BBR-treated group was significantly elevated, indicating that BBR plays an important role in the maturation of new vessels.

Our studies provided evidence from different aspects that BBR could promote the increase of cerebral blood perfusion in $3 \times \mathrm{Tg} \mathrm{AD}$ mice. It is indicated that berberine promoted angiogenesis, which improved blood perfusion of brain tissue. The underlying mechanisms of how BBR improves cognitive impairment and alleviates neuronal loss could be numerous, which include decreasing $\mathrm{A} \beta$ accumulation, accelerating angiogenesis, and recovering cerebral blood perfusion.

\section{Conclusions}

The BBR can clearly improve the learning and memory abilities of the AD model mice. The BBR's protection on AD may be connected with promoting CBF recovery and repressing the pathology of AD. Taking all these results into consideration, it could be concluded that BBR could promote angiogenesis, accelerate the elimination of toxic substances such as $A B$ in the tissue structure around microvessels, and inhibit the apoptosis of nerve cells. These results suggest that BBR could have the potential for the therapy of Alzheimer's disease through promoting CBF recovery.

Author Contributions: Methodology, C.Y.; software, Y.L.; formal analysis, Y.C. and X.Z.; data curation, Y.X. and Y.S.; writing-original draft preparation, C.Y.; writing-review and editing, M.H.; supervision, H.C.; project administration, M.H. All authors have read and agreed to the published version of the manuscript.

Funding: This work was supported by the National Natural Science Foundation of China (No. 81901422). Shenzhen Bureau of Science, Technology and Information (Nos. JCYJ20180307151246821), and the Project of Traditional Chinese Medicine of Guangming District (No. GM2019020029, No. GM2019020028), and Sanming Project of Medicine in Shenzhen (No. SZSM 201911003).

Institutional Review Board Statement: The procedures utilized in this study were conducted strictly according to the institutional guideline of experimental animal use in Sun Yat-sen University. The protocol was approved by the Animal Ethical and Welfare Committee of Sun Yat-sen University (Permit Number: SYXK2016-0112).

Informed Consent Statement: Not applicable.

Data Availability Statement: All data generated or analysed during this study are included in this published article.

Conflicts of Interest: The authors declare no conflict of interest. 


\section{References}

1. Hampel, H.; Vergallo, A.; Afshar, M.; Akman-Anderson, L.; Arenas, J.; Benda, N.; Batrla, R.; Broich, K.; Caraci, F.; Cuello, A.C.; et al. Blood-based systems biology biomarkers for next-generation clinical trials in Alzheimer's disease. Dialogues Clin. Neurosci. 2019, 21, 177-191. [PubMed]

2. Adav, S.S.; Sze, S.K. Hypoxia-Induced Degenerative Protein Modifications Associated with Aging and Age-Associated Disorders. Aging Dis. 2020, 11, 341-364. [CrossRef]

3. Kalaria, R.N. Neuropathological diagnosis of vascular cognitive impairment and vascular dementia with implications for Alzheimer's disease. Acta Neuropathol. 2016, 131, 659-685. [CrossRef]

4. Kandasamy, M.; Anusuyadevi, M.; Aigner, K.M.; Unger, M.S.; Kniewallner, K.M.; de Sousa, D.M.B.; Altendorfer, B.; Mrowetz, H.; Bogdahn, U.; Aigner, L. TGF- $\beta$ Signaling: A Therapeutic Target to Reinstate Regenerative Plasticity in Vascular Dementia? Aging Dis. 2020, 11, 828-850. [CrossRef] [PubMed]

5. Archer, H.A.; Smailagic, N.; John, C.; Holmes, R.B.; Takwoingi, Y.; Coulthard, E.J.; Cullum, S. Regional cerebral blood flow single photon emission computed tomography for detection of Frontotemporal dementia in people with suspected dementia. Cochrane Database Syst. Rev. 2015, 23, CD010896. [CrossRef] [PubMed]

6. Poon, C.H.; Wang, Y.; Fung, M.-L.; Zhang, C.; Lim, L.W. Rodent Models of Amyloid-Beta Feature of Alzheimer's Disease: Development and Potential Treatment Implications. Aging Dis. 2020, 11, 1235-1259. [CrossRef]

7. Bailey, T.L.; Rivara, C.B.; Rocher, A.B.; Hof, P.R. The nature and effects of cortical microvascular pathology in aging and Alzheimer's disease. Neurol. Res. 2004, 26, 573-578. [CrossRef]

8. Lorius, N.; Locascio, J.J.; Rentz, D.M.; Johnson, K.A.; Sperling, R.A.; Viswanathan, A.; Marshall, G.A.; Alzheimer's Disease Neuroimaging Initiative. Vascular disease and risk factors are associated with cognitive decline in the alzheimer disease spectrum. Alzheimer Dis. Assoc. Disord. 2015, 29, 18-25. [CrossRef]

9. Prohovnik, I.; Mayeux, R.; Sackeim, H.A.; Smith, G.; Stern, Y.; Alderson, P.O. Cerebral perfusion as a diagnostic marker of early Alzheimer's disease. Neurology 1988, 38, 931-937. [CrossRef]

10. Pluta, R.; Furmaga-Jabłońska, W.; Maciejewski, R.; Ułamek-Kozioł, M.; Jabłoński, M. Brain ischemia activates $\beta$ - and $\gamma$-secretase cleavage of amyloid precursor protein: Significancein sporadic Alzheimer's disease. Mol. Neurobiol. 2013, 47, 425-434. [CrossRef]

11. You, Y.; Perkins, A.; Cisternas, P.; Muñoz, B.; Taylor, X.; You, Y.; Garringer, H.J.; Oblak, A.L.; Atwood, B.K.; Vidal, R.; et al. Tau as a mediator of neurotoxicity associated to cerebral amyloid angiopathy. Acta Neuropathol. Commun. 2019, 7, 26. [CrossRef] [PubMed]

12. Jin, K. A Microcirculatory Theory of Aging. Aging Dis. 2019, 10, 676-683. [CrossRef]

13. Ochalek, A.; Mihalik, B.; Avci, H.X.; Chandrasekaran, A.; Téglási, A.; Bock, I.; Giudice, M.L.; Táncos, Z.; Molnár, K.; László, L.; et al. Neurons derived from sporadic Alzheimer's disease iPSCs reveal elevated TAU hyperphosphorylation, increased amyloid levels, and GSK3B activation. Alzheimers Res. Ther. 2017, 9, 90. [CrossRef]

14. Barrera-Ocampo, A.; Lopera, F. Amyloid-beta immunotherapy: The hope for Alzheimer disease? Colomb. Med. 2016, 47, $203-212$. [CrossRef]

15. Kulkarni, S.K.; Dhir, A. Berberine: A plant alkaloid with therapeutic potential for central nervous system disorders. Phytother. Res. 2010, 24, 317-324. [CrossRef]

16. Huang, M.; Chen, S.; Liang, Y.; Guo, Y. The role of berberine in the multi-target treatment of senile dementia. Curr. Top. Med. Chem. 2016, 16, 867-873. [CrossRef] [PubMed]

17. Oddo, S.; Caccamo, A.; Shepherd, J.D.; Murphy, M.P.; Golde, T.E.; Kayed, R.; Kayed, R.; Metherate, R.; Mattson, M.P.; Akbari, Y.; et al. Triple-transgenic model of Alzheimer's disease with plaques and tangles: Intracellular Abeta and synaptic dysfunction. Neuron 2003, 39, 409-421. [CrossRef]

18. Kong, W.; Wei, J.; Abidi, P.; Lin, M.; Inaba, S.; Li, C.; Wang, Y.; Wang, Z.; Si, S.; Pan, H.; et al. Berberine is a novel cholesterollowering drug working through a unique mechanism distinct from statins. Nat. Med. 2004, 10, 1344-1351. [CrossRef]

19. Jing, Z.; Shi, C.; Zhu, L.; Xiang, Y.; Chen, P.; Xiong, Z.; Li, W.; Ruan, Y.; Huang, L. Chroniccerebral hypoperfusion induces vascular plasticity and hemodynamics but also neuronal degeneration and cognitive impairment. J. Cereb. Blood Flow Metab. 2015, 35, 1249-1259. [CrossRef]

20. Mueller, S.C.; Temple, V.; Oh, E.; VanRyzin, C.; Williams, A.; Cornwell, B.; Grillon, C.; Pine, D.S.; Ernst, M.; Merke, D.P. Early and rogen exposure modulates spatial cognition in congenital adrenal hyperplasia (CAH). Psychoneuroendocrinology 2008, 33, 973-980. [CrossRef]

21. Vorhees, C.V.; Williams, M.T. Morris water maze: Procedures for assessing spatial and related forms of learning and memory. Nat. Protoc. 2006, 1, 848-858. [CrossRef]

22. de la Torre, J.C. Is Alzheimer's disease a neurodegenerative or a vascular disorder? Data, dogma, and dialectics. Lancet Neurol. 2004, 3, 184-190. [CrossRef]

23. Ni, Y.-Q.; Lin, X.; Zhan, J.-K.; Liu, Y.-S. Roles and Functions of Exosomal Non-coding RNAs in Vascular Aging. Aging Dis. 2020, 11, 164-178. [CrossRef] [PubMed]

24. Kogure, D.; Matsuda, H.; Ohnishi, T.; Asada, T.; Uno, M.; Kunihiro, T.; Nakano, S.; Takasaki, M. Longitudinal evaluation of early Alzheimer's disease using brain perfusion SPECT. J. Nucl. Med. 2000, 41, 1155-1162. [PubMed]

25. Rodriguez, G.; Vitali, P.; Calvini, P.; Bordoni, C.; Girtler, N.; Taddei, G.; Mariani, G.; Nobili, F. Hippocampal perfusion in mild Alzheimer's disease. Psychiatry Res. 2000, 100, 65-74. [CrossRef] 
26. Jack, C.R., Jr.; Petersen, R.C.; Xu, Y.C.; O’Brien, P.C.; Smith, G.E.; Ivnik, R.J.; Boeve, B.F.; Waring, S.C.; Tangalos, E.G.; Kokmen, E. Prediction of AD with MRI-based hippocampal volume in mild cognitive impairment. Neurology 1999, 52, 1397-1403. [CrossRef]

27. Cai, Z.; Liu, Z.; Xiao, M.; Wang, C.; Tian, F. Chronic Cerebral Hypoperfusion Promotes Amyloid-Beta Pathogenesis via Activating $\beta / \gamma$-Secretases. Neurochem. Res. 2017, 42, 3446-3455. [CrossRef]

28. Aski, M.L.; Rezvani, M.E.; Khaksari, M.; Hafizi, Z.; Pirmoradi, Z.; Niknazar, S.; Mehrjerdi, F.Z. Neuroprotective effect of berberine chloride on cognitive impairment and hippocampal damage in experimental model of vascular dementia. Iran J. Basic Med. Sci. 2018, 21, 53-58.

29. Jia, Y.; Wang, N.; Zhang, Y.; Xue, D.; Lou, H.; Liu, X. Alteration in the Function and Expression of SLC and ABC Transporters in the Neurovascular Unit in Alzheimer's Disease and the Clinical Significance. Aging Dis. 2020, 11, 390-404. [CrossRef]

30. Li, W.; Kui, L.; Demetrios, T.; Gong, X.; Tang, M. A Glimmer of Hope: Maintain Mitochondrial Homeostasis to Mitigate Alzheimer's Disease. Aging Dis. 2020, 11, 1260-1275. [CrossRef]

31. Do, T.M.; Alata, W.; Dodacki, A.; Traversy, M.-T.; Chacun, H.; Pradier, L.; Scherrmann, J.-M.; Farinotti, R.; Calon, F.; Bourasset, F. Altered cerebral vascular volumes and solute transport at the blood-brain barriers of two transgenic mouse models of Alzheimer's disease. Neuropharmacology 2014, 81, 311-317. [CrossRef]

32. Salminen, A.; Kauppinen, A.; Kaarniranta, K. Hypoxia/ischemia activate processing of Amyloid Precursor Protein: Impact of vascular dysfunction in the pathogenesis of Alzheimer's disease. J. Neurochem. 2017, 140, 536-549. [CrossRef] [PubMed]

33. Crouch, E.E.; Doetsch, F. FACS isolation of endothelial cells and pericytes from mouse brain microregions. Nat. Protoc. 2018, 13, 738-751. [CrossRef] [PubMed]

34. Solerte, S.B.; Ferrari, E.; Cuzzoni, G.; Locatelli, E.; Giustina, A.; Zamboni, M.; Schifino, N.; Rondanelli, M.; Gazzaruso, C.; Fioravanti, M. Decreased release of the angiogenic peptide vascular endothelial growth factor in Alzheimer'sdisease: Recovering effect with insulin and DHEA sulfate. Dement. Geriatr. Cogn. Disord. 2005, 19, 1-10. [CrossRef]

35. Chakraborty, A.; Chatterjee, M.; Twaalfhoven, H.; Del Campo Milan, M.; Teunissen, C.E.; Scheltens, P.; Fontijn, R.D.; van Der Flier, W.M.; de Vries, H.E. Vascular Endothelial Growth Factor remains unchanged in cerebrospinal fluid of patients with Alzheimer's disease and vascular dementia. Alzheimers Res. Ther. 2018, 10, 58. [CrossRef]

36. Hozzein, W.N.; Badr, G.; Badr, B.M.; Allam, A.; Ghamdi, A.A.; Al-Wadaan, M.A.; Al-Waili, N.S. Bee venom improves diabetic wound healing by protecting functional macrophages from apoptosis and enhancing Nrf2, Ang-1 and Tie-2 signaling. Mol. Immunol. 2018, 103, 322-335. [CrossRef]

37. Bitto, A.; Minutoli, L.; Galeano, M.R.; Altavilla, D.; Polito, F.; Fiumara, T.; Calò, M.; Lo Cascio, P.; Zentilin, L.; Giacca, M.; et al. Angiopoietin-1 gene transfer improves impaired wound healing in genetically diabetic micewithout increasing VEGF expression. Clin. Sci. 2008, 114, 707-718. [CrossRef]

38. Tillet, E.; Vittet, D.; Féraud, O.; Moore, R.; Kemler, R.; Huber, P. N-cadherin deficiency impairs pericyte recruitment, and not endothelial differentiation or sprouting, in embryonic stem cell-derived angiogenesis. Exp. Cell Res. 2005, 310, 392-400. [CrossRef]

39. Lelièvre, E.C.; Plestant, C.; Boscher, C.; Wolff, E.; Mège, R.M.; Birbes, H. N-cadherin mediates neuronal cell survival through Bim down-regulation. PLoS ONE 2012, 7, e33206. [CrossRef] 